\title{
ON HEIGHTS IN NUMBER FIELDS ${ }^{1}$
}

\author{
BY S. SCHANUEL
}

Communicated by Felix Browder, May 14, 1963

Let $K$ be a number field, of degree $N$ over $\boldsymbol{Q}$.

Let $S_{\infty}$ be the set of archimedean absolute values of $K$, normalized to extend the ordinary absolute value on $Q$. For $x \in K^{*}, v \in S_{\infty}$, put $\|x\|_{v}=|x|_{v}^{N_{v}}$, where $N_{v}$ is the local degree $\left[K_{v}: Q_{v}\right]$, so $N_{v}=1$ or 2 . For $X=\left(X_{1}, \cdots, X_{m}\right) \in K^{m}$, put $\|X\|_{v}=\sup \left\|X_{i}\right\|_{v}, H_{\infty}(X)$ $=\prod_{v \in S_{\infty}}\|X\|_{v}$; and let $[X]$ denote the fractional ideal generated by $X_{1}, \cdots, X_{m}$. Then the height of $X$ is $N[X]^{-1} H_{\infty}(X)$.

The class of a point in projective space $P^{m-1}(K)$ is the class (modulo principal ideals) of the fractional ideal generated by homogeneous coordinates for $X$.

THEOREM 1. The number of points in $P^{m-1}(K)$ of a given class, with height at most $B$, is

$$
\frac{\kappa_{m}}{\zeta_{K}(m)} B^{m}+O\left(B^{m-1 / N}\right)
$$

where

$$
\kappa_{m}=\left(\frac{2^{r_{1}}(2 \pi)^{r_{2}}}{\sqrt{ } d}\right)^{m} \frac{R}{w} m^{r} ;
$$

except that for $m=2, N=1$, the error term is to be replaced by $O(B \log B)$.

The notation is standard (cf. [1]). For a discussion of the setting of the problem, see [2, Chapter III]. The burden of the proof is carried by Theorem 2 .

An $S_{\infty}$-divisor, or simply divisor on $K$ is a pair $\mathfrak{b}=(\mathfrak{a}, B)$, where $\mathfrak{a}$ is a nonzero fractional ideal and $B$ is a positive real number. The norm of $\delta$ is $\|\delta\|=N a^{-1} B$. Map $K^{m}-0^{m}$ to the group of divisors by $\mathfrak{b}_{X}=\left([X], H_{\infty}(X)\right)$. For $m=1$, this is a homomorphism, with kernel $U$ (the group of units of $K$ ) and image the principal divisors.

Let $U$ act on $K^{m}$ by componentwise multiplication. Then associated to any divisor $\mathfrak{D}$ is an $S_{\infty}$-parallelotope $L_{m}(\mathfrak{D}) \subset\left(K^{m}-0^{m}\right) / U$; it is the set of all orbits $U X$ for which $\mathfrak{D}_{X} \leqq \mathfrak{d}$, that is: $[X] \subset \mathfrak{a}, H_{\infty}(X) \leqq B$. Similarly, the restricted $S_{\infty}$-parallelotope $L_{m}^{\prime}(\mathfrak{D})$ is the set of all $U X$ for which $[X]=\mathfrak{a}, H_{\infty}(X) \leqq B$. Let $\lambda_{m}, \lambda_{m}^{\prime}$ be the cardinalities of $L_{m}$, $L_{m}^{\prime}$.

\footnotetext{
${ }^{1}$ Details and related results will appear in a forthcoming paper.
} 
REMARK 1. $\lambda_{m}(\delta), \lambda_{m}^{\prime}(\delta),\|\delta\|$ depend only on the class of $\delta$ modulo principal divisors.

REMARK 2. $\lambda_{m}(\mathfrak{D})=\lambda_{m}^{\prime}(\mathfrak{d})=0$ for $\|\mathfrak{d}\|<1$.

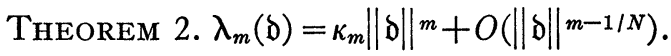

One may restrict $\mathfrak{d}$ to range over divisors $\left(\mathfrak{a}_{i}, B\right)$, where $\mathfrak{a}_{i}$ are representatives for the ideal classes, by Remark 1 ; thus it suffices to consider divisors with $a$ fixed. For $m=1, L_{1}(a, B)$ is the set of principal ideals contained in $\mathfrak{a}$, of norm at most $B$. Hence Theorem 2 reduces in this case to a classical theorem due to Dedekind and Weber [3].

The reduction of Theorem 1 to Theorem 2 is based on two easy observations. First, there is a bijection from $L_{m}^{\prime}(\mathfrak{a}, B N \mathfrak{a})$ to the set of points of $P^{m-1}(K)$ of class $\mathrm{Cl}(\mathfrak{a})$, by $U X \rightarrow K^{*} X$, so that the problem is to estimate $\lambda_{m}^{\prime}$. Second, $L_{m}(\mathfrak{a}, B)=\bigcup L_{m}^{\prime}(\mathfrak{a} \mathfrak{b}, B)$, the (disjoint) union extending over all integral ideals $\mathfrak{b}$. Thus

$$
\lambda_{m}(\mathfrak{a}, B)=\sum \lambda_{m}^{\prime}(\mathfrak{a} \mathfrak{b}, B) .
$$

(The sum is finite, since $\lambda_{m}^{\prime}(\mathfrak{a} \mathfrak{b}, B)=0$ for $N \mathfrak{b}>B N \mathfrak{a}^{-1}$, by Remark 2.) $A$ variant of the Möbius inversion formula gives

$$
\lambda_{m}^{\prime}(\mathfrak{a}, B)=\sum \mu(\mathfrak{b}) \lambda_{m}(\mathfrak{a} \mathfrak{b}, B)
$$

By Theorem 2, the sum on the right is

$$
\sum_{\mu(\mathfrak{b})}\left(\kappa_{m}\left(\frac{\|\mathfrak{d}\|}{N \mathfrak{b}}\right)^{m}+O\left(\left(\frac{\|\mathfrak{d}\|}{N \mathfrak{b}}\right)^{m-1 / N}\right)\right),
$$

summed over all integral $\mathfrak{b}$ with $N \mathfrak{b} \leqq\|\mathfrak{b}\|=B N \mathfrak{a}^{-1}$. The first term contributes

$$
\kappa_{m}\|\delta\|^{m}\left(\frac{1}{\zeta_{K}(m)}+O\left(\|\delta\|^{1-m}\right)\right)
$$

and the second is easily estimated to yield Theorem 1.

\section{BIBLIOGRAPHY}

1. Erich Hecke, Vorlesungen über die Theorie der algebraischen Zahlen, AkademieVerlag, Leipzig, 1923.

2. Serge Lang, Diophantine geometry, Interscience, New York, 1962.

3. Heinrich Weber, Lehrbuch der Algebra, Band II, Absch. 20, 21, Vieweg, Braunschweig, 1899.

Columbia University 